\title{
Caveolins; An Assailant or An Ally of Various Cellular Disorders
}

\author{
Authors \\ Ritesh Kumar Srivastav' ${ }^{1}$ Tarique Mahmood Ansari², Mahesh Prasad1', Vishal Kumar Vishwakarma ${ }^{3}$ \\ Paramdeep Bagga², Farogh Ahsan²
}

\section{Affiliations}

1 Faculty of Pharmacy, Kamla Nehru Institute of Management and Technology, Sultanpur, India

2 Faculty of Pharmacy, Integral University, Lucknow, Uttar Pradesh, India

3 Department of Pharmacology, R.R.S College of Pharmacy, Amethi, Uttar Pradesh, India

Key words

caveolins, cardiovascular disorder, diabetic, ischaemic preconditioning, nitric oxide, cell growth

$\begin{array}{ll}\text { received } & 01.01 .2019 \\ \text { accepted } & 01.02 .2019\end{array}$

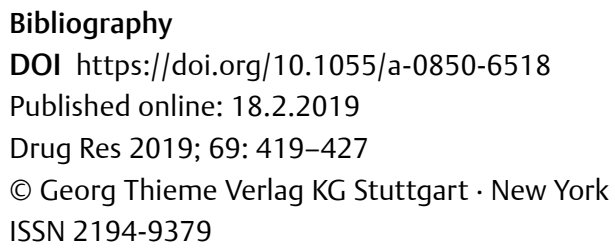

\section{Correspondence}

Tarique Mahmood Ansari

Faculty of Pharmacy

Integral University,

Lucknow

Uttar Pradesh-226021

India

Tel.: + 91/991/8681 701

ansaritarique7886@gmail.com

\section{ABSTRACT}

Caveolae have impressive morphological highlights of the cytomembrane of mammalian cells which involve in wide diversity of cellular functions involving signaling pathways and cholesterol hastening. Caveolin proteins possess a 'scaffolding' domain which for caveolin-1 and caveolin-3 appear to act a dominant role in signal regulation through caveolae. Caveolin-1 is treated to be protein in the cytomembrane entrapped with caveolae in endothelial cells and vascular smooth muscle cells which diminish nitric oxide (NO) by fill up the calcium/ calmodulin $\left(\mathrm{Ca}^{2+} / \mathrm{CaM}\right)$ confining point of endothelial nitric oxide synthase (eNOS), decrease NO generation produce endothelial dysfunction and atherosclerotic injury development. It is a cholesterol-binding layer protein associated with cell cholesterol transport and also shows cardioprotective action through ischemic preconditioning (IPC) in diabetic and postmenopausal rat heart. Additionally it is ensnared in the procedures of tumorigenesis, prostate disease, and inflammation. The present study in the paper is to explore the structural functionalities of caveolins and their contributory role in CVS disorders and various other diseases.

\section{Introduction}

Caveolin is a originally protein made up of caveolae in adipocytes and the caveolae themselves significant role in lipid uptake and supply to the lipid globule in these cells. Caveolae provide a centralactvity in the growth factor and signaling of hormones. Caveolins are transmembrane proteins possessing intracellular domain that undergo extensive oligomerization to form membrane complexes known as caveolae [1]. In 1955, Yamada [2] given the descriptive name "caveolae" which is Latin for little caves. In 1953, Caveolae were primarily represent by the electron microscopist by Palade [3] and are plentiful in many vertebrate cells, specifically in endothelial cells and adipocytes where they may represent $30-70 \%$ of the total surface area of plasma membrane. Caveolae however, are not a com- mon character of all cell as they are not totally present in neurons. Like lipid raft, caveolae are partially described and enhanced in sphingolipids and cholesterol and furthermore take an interest in signal transduction processes. Caveolae are invaginations of the plasma layer with strikingly characterized vessels like structure [4].

\section{Caveolins and Caveolae}

Nonidentical types of cell have distinctive densities of caveolae in their cytomembrane. Mean, about half of the surface plasmalemma of adipocyte contains caveolae [5], while just $5 \%$ of fibroblast cytomembrane is build up of caveolae [6]. A noteworthy characterizing and basic part of caveolae is the presence of proteins like caveolin [7], junction that involved directly with cholesterol. These cholesterol is a leading part of caveolae, and exhaustion from ca- 
veolae makes them linear shape and can decrease or wipe out caveolae at the cell. It is proposed that a basic amount of these cholesterol are essential for the development and upkeep of caveolae; in any case, the correct connection between the cellular cholesterol and caveolins isn't yet unknown. The oligomerization of caveolins and collaboration with cholesterol supports produce the invaginated organize of this substances [8].

Three type of caveolin-encoding gene i.e CAV1, CAV2, and CAV3 are translated to exhibit 6 known subtypes of the protein [9]. Caveolins have 18-20 kDa proteins and an also have extraordinary hairpin structure with cytoplasmic $\mathrm{N}$-and $\mathrm{C}$-ends. Cav- 1 is communicated in totally cell composes including epithelial, adipocytes, smooth muscle and fibroblast cells [10]. Cav-3 is particularly communicated in cardiovascular muscle and additionally skeletal and smooth muscle [11]. Cavs- 1 and 3 are communicated autonomously of each other and are required for the development of caveolae, and Cav-2 partners with Cav-1 or 3 however isn't engaged with caveolae arrangement [12].

\section{Localization of caveolin}

Caveolin is the caveolar membrane protein which invaginated on the plasma layer that fills in as signaling stage for a considerable lot of G-protein coupled receptors [13]. Caveolin-1 gives off an impression of being embedded co-translationally into the ER membrane along its $\mathrm{C}$-and $\mathrm{N}$-terminal segments in the cytoplasm. It at that point is fused into vesicles that rotate to the Golgi body in a stage that desires amino acids 66-70 [14]. Inside the Golgi body cav-1 oligomerizes and winds up cleanser insoluble [15]. Oligomerization relies upon amino acids $91-100$ and $135-140$. Vesicular transport to the cell surface depends both on the capacity of the particle to oligomerize and on amino acids $71-80$. When it achieves the cell surface, apparently cav-1 become consolidated into working caveolae that disguise and reuse. At some phase in caveolae disguise cycle, we accept cav-1 can enter the cytoplasm of the cell as a soluble protein mantained in a lipid molecule. Corrected amino acids in the protein that maintain this progression are not clear but rather may rely upon palmitoylation of cysteines 133,143 , and 156 [16].

\section{Contribution of caveolin}

Polymerase I and transcript release factor (PTRF)

In year 2001 a $60 \mathrm{kD}$ caveolae particular protein was distinguished on the cytosolic face of the cytomembrane. It was recognized utilizing immunogold marking of epitopes on cytosolic surface of plasma membrane in adipocyte cells utilizing a monoclonal antibody [17]. This protein ended up being indistinguishable to a formerly recognized protein called PTRF [18] or then again called binding factor of type-1 collagen promote [19]. Afterward, it was exhibited that this protein colocalizes with cav-1 in human being adipocytes [20].

The correct part of this protein isn't known; in any case, it is thought to know an auxiliary and conceivably a useful part in caveolae. It is communicated profoundly in lungs, heart, colon and adipocytes however it is additionally communicated in the kidney, testis thymus, and spleen and has been appeared to be feebly communicated in liver and cerebrum [20].

\section{Cholesterol}

The real segment of lipid rafts and caveolae is cholesterol and re-localization of caveolae cholesterol of free cholesterol significantly diminishes the quantity of caveolae [21]. The present information recommend that there is a basic cholesterol level required for the development and upkeep of caveolae yet a reasonable comprehension of this rational pair has not been revealed. Expanded, levels of lowdensity lipoprotein (LDL) cholesterol prompt expanded arrangement of caveolae in smooth muscle and fibroblasts cells [22]. While an expansion in pre-HDL prompts diminished development of caveolae [23]. Besides, cholesterol levels in the cell don't stay like rigid and cholesterol continually flows into and out of caveolae [24].

\section{Signal transduction}

It is presently entrenched that caveolae partition and manage a wide assortment of signaling pathways [25] despite the fact that a little minority has questioned the part of caveolae in signaling [26]. In any case, it is imperative to comprehend that a significant number of the signaling pathways in caveolae likewise work outside of caveolae which in all probability clarifies why caveolin null mice are as yet reasonable.

\section{Endothelial nitric oxide synthase}

One manner by which caveolae can impact oxidative stress which may prompt cardiac disease is through the control of eNOS [27]. Nitric oxide produced from eNOS is mainly in directing circulatory system, angiogenesis, vascular smooth muscle cell expansion and inhibition of platelet agreegation [28]. A few investigations have shown that both lacking levels of nitric oxide (NO) and in addition abundance measures of nitric oxide can be harmful [29]. Like caveolae, eNOS is mainly expressed in endothelial cells and in addition in heart myocytes [30]. The mechanism include the generation of NO from eNOS or different NOS isoforms is through a response where arginine is oxidized to citrulline discharging NO. Homodimers of NOS tie to cofactors, for example, NADPH, FAD and FMN, and Calmodulin which show a basic and useful part. The focusing of eNOS to caveolae is through change by palmitoylation and Nmyristoylation ( $\triangleright$ Fig. 1). Once connected with caveolae, eNOS associates with the platform area of cav-1 in endothelial cell and cav-3 in cardiovascular myocytes [31]. The cooperation of caveolin with eNOS assumes an inhibitory role and it is just upon the arrival of eNOS from caveolin that the catalyst can be completely initiated.

\section{Caveolin as an objective for an assortment of diseases}

Cav-1,2,3 and cav-1/-3 double knockout KO mice have been created (Cav-KO mice), albeit practical and prolific. Cav-2 KO mice stop simple expression of caveolae. Cav-1 KO mice additionally need cav-2 and caveolae and create cardiomyopathy, pulmonary blood vessel hypertension, redesigning in the lung and right ventricular hypertrophy. It is misty if the phenotypes of cav KO mice result specifically or in a roundabout way from the loss of presence of caveolae and caveolins. It will hold any importance with decide the phenotypes in animals that have spatially and temporally particular loss of presence of individual caveolins [32]. 


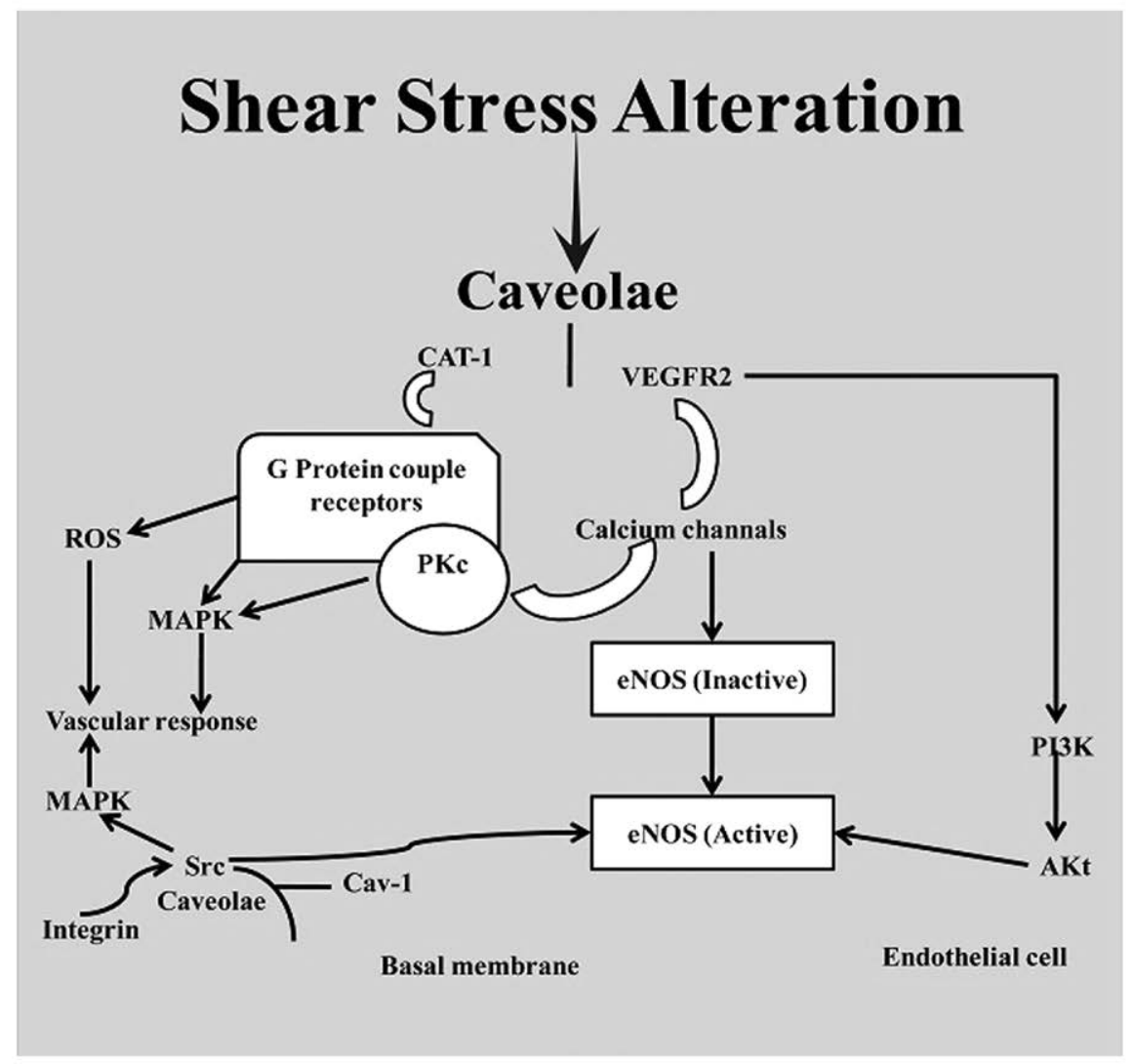

- Fig. 1 Cav-1 controlled signaling pathways in endothelial cells. Caveolae and Cav-1 seem, by all accounts, to be engaged with a portion of the most punctual advances related with the identification of modified shear pressure conditions in veins. Furthermore, the vascular reaction is incited through caveolae Cav-1 assumes imperative parts in managing eNOS work. In unstimulated cells, eNOS is kept up in a latent state through its relationship with Cav-1. Upon incitement (e. g., shear pressure), Cav-1 and caveolae may take into account the best possible association of different flag transduction pathways or arrange the diverse administrative proteins fundamental for fast and effective eNOS enactment. Calcium channels and the arginine transporter CAT- 1 are restricted to caveolae and may along these lines take into account productive eNOS actuation (Ca2 + section and separation from Cav-1 within the sight of calmodulin) and accessibility of substrate. Advance actuation is additionally conceivable by means of the PI3K and Akt signaling pathways, which upgrade NO creation following eNOS phosphorylation. Enactment of the VEGFR2 signaling pathway is basic for PI3K/Akt initiation, however different pathways appear to likewise intervene MAPK actuation (ERK1/2 specifically). These pathways likewise include $G$ protein- coupled receptors (GPCRs) and G proteins. Shear pressure is related with expanded oxidative pressure conditions that prompt the creation of ROS. Furthermore, shear pressure may influence EC cooperations with extracellular lattice proteins. This may thus initiate integrin-intervened signaling pathways by means of caveolae. Src may likewise straightforwardly change eNOS action by prompting tyrosine phosphorylation.

\section{Role in cardiovascular}

Endothelial abnormality as showed by a enhence of NO mediated vasodilation, is perceived to be an essential anomaly in the beginning of hypertension, coronary artery disease and atherosclerosis. Endothelial abnormality in link with metabolic variations is typically produced by a combination of diminished generation and expanded obliteration of NO prompting to a down in NO bioavailability [33]. NO generation in response of different variables, for example, expanded shear stress, is mediated by eNOS, which is constitutively communicated in endothelial cells and is tightly controlled by various regulatory proteins and membrane bound receptors under physiological conditions [34]. Increments in eNOS and caveolin connection, as may happen with hyperlipidemia, reduce NO release and stimulate endothelial damage and atherosclerotic injury development ( $\triangleright$ Fig. 2). This procedure is mediated by expanded lipoprotein trafficking over the vascular endothelium. The pharma- cological role of Cav-1 in the control of eNOS activation and gave confirm that the connection of eNOS and Cav- 1 could be a better target site for the direction of cardiac disorder [35]. In other words it is important to create clinically and safely powerful modulators of Cav-1/eNOS connection in vascular and entire body insulin homeostasis maintain in the population with T2D [36].

\section{Role in kidney}

The function of caveolae in the kidney, where prior investigations depicted the nearness of Cav- 1 and caveolae in the distal renal epithelia and vasculature [37]. Phenotyping of Cav 1-defcient mice uncovered direct urinary loss of magnesium, calcium, and potassium, proposing that caveolae may assume a part in renal treatment of these electrolytes. This effects are accepted to rely upon function interaction of Cav-1 with potassium transport proteins and basolateral calcium [38]. An ongoing report in vasopressin-defcient Brattleboro 
rats with main point of diabetes insipidus (DI) reported that a part for Cav-1 in the urinary concentraction process; incitement of DI rats with vasopressin $\mathrm{V} 2$ receptor agonist like desmopressin instigated a managed apical translocation of Cav-1 in target cells of collecting ducts. The practical signifcance of caveolae for kidney reabsorption of water and salt, be that as it may, stayed to be illustrated further [39]. Inhibitory impacts of caveolae on activity of NOS isoforms have been accounted for in various past examinations [40].

\section{Role in cancer cell}

Cancer is a multiple development procedure, in which enactment of oncogenes or supression of tumor suppressor gene are wonders assuming critical parts [41]. Cance is to a great extent described by the uncontrolled development of irregular cells getting away from the immune system, protection from apoptosis, and the capacity to trigger metastasis and angiogenesis. Molecular aleration responsible for tumorigenesis and malignant cell are caused by both hereditary transformations and epigenetic mechanism identified with changes in gene expression that are essentially intervened by histone and DNA methylation modification. Disturbance of the epigenome is additionally a key mechanism in cell growth where direction of tumor growth is regulated by a harmony amongst tumor suppressors and oncogenes. Many studies have revealed the significance of Cav-1 during the multistep of tumor progession and its appearance was distinguished in changed type of tumors [42]. In a many reports, Cav-1 presence was observed to be related with tumor size, stage and grade which demonstrated its relationship with the advancement of growths of cell. Nonetheless, it is apparent that Cav-1 additionally goes about as a tumor suppressor [43].

Among these, Cav-1 presence was found to connect with protection from ionizing radiation [44]. Cav-1, by means of its scaffolding domain, interfaces with an assortment of important proteins that are shown in lipid raft and caveolar membrane, for example, platelet derived development factor receptor, receptor tyrosine kinases, epidermal development factor receptor, eNOS, proteins engaged with serine/threonine kinases, calcium transport, integrins, nerve development factor, phospholipases, GPCR and Src family Kinases [45]. Such collaborations in this way create multiple signaling stages which encourage the signaling cascades that add to malignancy control. Such connections along these lines create multiple signaling platform which encourage the signaling cascades that add to cell growth regulation. The level of intracellular Cav-1 expression is high related with metastatic progression of numerous type of malignancies, for example, prostate [46], lung [47], kidney [48] and esophageal cancer [49].

Role in insulin signal

In spite of the fact that caveolin may downregulated many enzyme species, there are a typical component among those enzyme that are liable to caveolin-mediated restraint [50]. The insulin receptor (IR) is proteins that have this motif, which exists in the kinase domain of the $\beta$-subunit of IR (amino acid residue 1193-1200 and WSFGVVLW). IR contains an endogenous tyrosine kinase inside its cytoplasmic domain. This kinase action is needed for transmission of downsregulated insulin signals, for example, cell proliferation and energy storage [51]. The IR tyrosine kinase is enhence by insulin binding, and completely actuated by the autophosphorylation of the regulatory area inside the kinase domain. Enactment of the IR kinase permits the tyrosine phosphorylation of intracellular substrates of the IR, for example, epidermal growth factor receptors, that additionally contain this theme, are potently repressed by caveolin [52]. Therefore, it was initially expected that caveolin additionally restrained IR and along these lines the insulin-initiated energy storage and cellular proliferation.

Different examinations have exhibited that caveolae furnish the TC10 complex associated with cellular microdomains for glucose transportation via Glut4. These discoveries propose that caveolin assumes an imperative part in insulin signal to keep up glucose digestion in intact animals. In any case, the role of caveolin in the insulin signal may vary from that in another transmembrane receptor signals [53].

\section{Role in airway}

Ciliary beating in airway epithelia cells can be stimulated by NO and acetylcholine [54]. Other than their release from nerve, they are additionally endogenously synthesized by the airway epithelium [55], in this manner giving an autocrine/paracrine route for the regulation of ciliary beat recurrence. Strikingly, the NO producing enzyme eNOS, cholinesterase that corrupts acetylcholine to choline and the high sffinity transporter (CHT1) that is engaged with consequent take-up of choline are situated in the apical part of rodent tracheal ciliated epithelial cells [56]. Since every one of these proteins are membrane related, these discoveries point towards the nearness of specialized membrane compartments, differ from the plasma membrane in ciliated cells, that could be associated with the regulation of ciliary beat frequency. In any case, how proteins can be caught in the apical region of ciliated cells is not know.

Cav-1 and cav- 2 both are communicated in ciliated cells of the trachea and large bronchi and are situated at the basolateral plasma membrane where they form caveolae [57]. Since the declaration of eNOS is additionally found in littler airway that are without cav- 1 , cav-2 and eNOS is found apically not basolaterally, cav- 1 and cav-2 can't be in responsible for the apical location of eNOS. Given the way that cav-3, aside from framing plasmalemmal caveolae, has as of late been found to shape microdomains in the sarcoplasmic reticulum of skeletal muscle [58], a membrane compartment that is distinct from the plasma membrane, we asked whether cav-3 could be the cav-isoform that keeps eNOS in a subplasmalemmal compartment in ciliated airway epithelial cells [59].

\section{Role in muscular disease}

Caveolin-3 shows to the muscle particular individual from the caveolin super-gene family [60] and has been shown to co-immunoprecipitate with dystrophin, proposing a biochemical association. Caveolae have a key part in the maintenance of plasma membrane integrity and in the procedures of vesicular trafficking and signals transduction. Mutation in the caveolin-3 gene prompt skeletal muscle pathology through various pathogenetic systems. Indeed, caveolin-3 inadequacy is related to sarcolemmal membrane changes, unorganized of skeletal muscle T-tubule system and disturbance of unmistakable cell-signaling pathways. To date, there have been 30 caveolin-3 mutation recognized in the human populace. Caveolin-3 absconds prompt 4 position skeletal muscle disease phenotypes: distal myopathy, appendage support musclar dystrophy, rippling muscle disease and hyperCKemia [61]. 


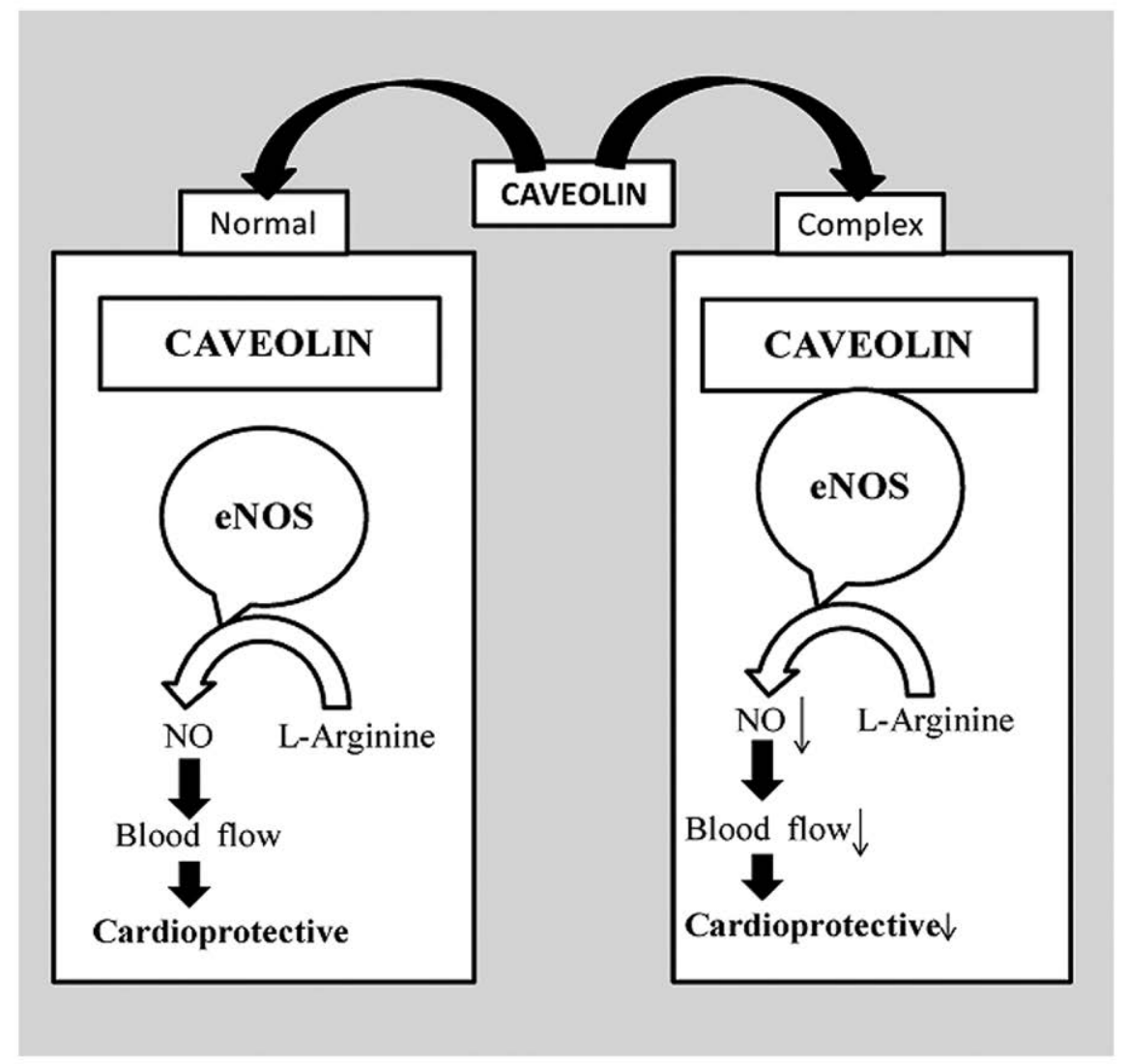

- Fig. 2 Role of heart Caveolin/eNOS in normal and disorder rats. eNOS: endothelial nitric oxide synthase; NO: nitric oxide.

\section{Role in diabetes}

Type II diabetes is a hereditarily heterogeneous disease influencing over $5 \%$ of the number of inhabitants in the Western world [62]. Every now and again, the primary recognizable variation from the norm recognized in people bound to create type II diabetes is insulin resistance, described by hyperinsulinemia and regularly hyperglycemia [63]. While the correct pathophysiological events responsible for the advancement of insulin resistance stay unknow, it is trusted that consolidated deformities in cell function and peripheral insulin activity are both imperative segments [64]. This fact has prompted the extraordinary investigation of the function of these organelles in adipocytes. It has now turned out to be obvious that effective insulin signaling in the adipocyte might be entirely subject to localiazation of no less than 2 insulin-responsive components to caveolae (insulin receptor and GLUT4), and also on a direct functional association between caveolin-1 and the insulin receptor ( Fig. 3) [65].

\section{Role in Alzehimer}

Alzheimer's disease (AD) is a neurodegenerative disorder described by alteration of cholesterol homeostasis in both the peripheral and central nervous system. Changes in cholesterol distribution have been appeared to be intently connected with the nearness of the apoc4 allele of the Apolipoprotein $\mathrm{E}$ (ApoE) in AD and control subjects [66]. The apoc4 allele (instead of the $\varepsilon 2$ and $\varepsilon 3$ alleles) is an outstanding danger factor for sporadic and familial late event $A D$
[67]. Patients with 2 \&4 alleles display a prior period of event [67], higher amyloid levels and amyloid plaque count [68], cerebrovascular amyloid [69] and a marked decrease in choline acetyltransferase [70] contrasted with non- $\varepsilon 4$ allele AD subjects.

Cholesterol assumes an essential part in cellular function and membrane compartmentalization. It is a basic part of lipid raft, thick lateral assemblies of cholesterol, and sphingolipids in the exoplasmic leaflet of the plasma membrane bilayer [71]. Caveolae, which are thought to be a particular type of raft, are omega-molded layer invaginations engaged with cell signaling, transcytosis, and in the control of cell cholesterol homeostasis [72]. Changes in cholesterol homeostasis related with either age, ApoE lack, or AD may modify the structure and function integrity of membrane microdomains. The expanded caveolin articulation saw in the brain of aged and ApoE knockout mice and all the more critically in AD patients' brains bolsters this suspicion. A dysregulated articulation of caveolin may be in charge of adjustments in caveolin-subordinate signaling transduction in cells. In addition, the high level of caveolin in cerebrum tissue of AD patients may give some insight to the component hidden the pathophysiology of AD [73].

Role in inflammation

Caveolins in immune and inflammatory reactions is simply starting to be explained. For instance, little interfering RNA knockdown of endothelial cav-1 impair the transcellular route of transendothelial migration of leukocytes, and mice hereditarily insufficient in 


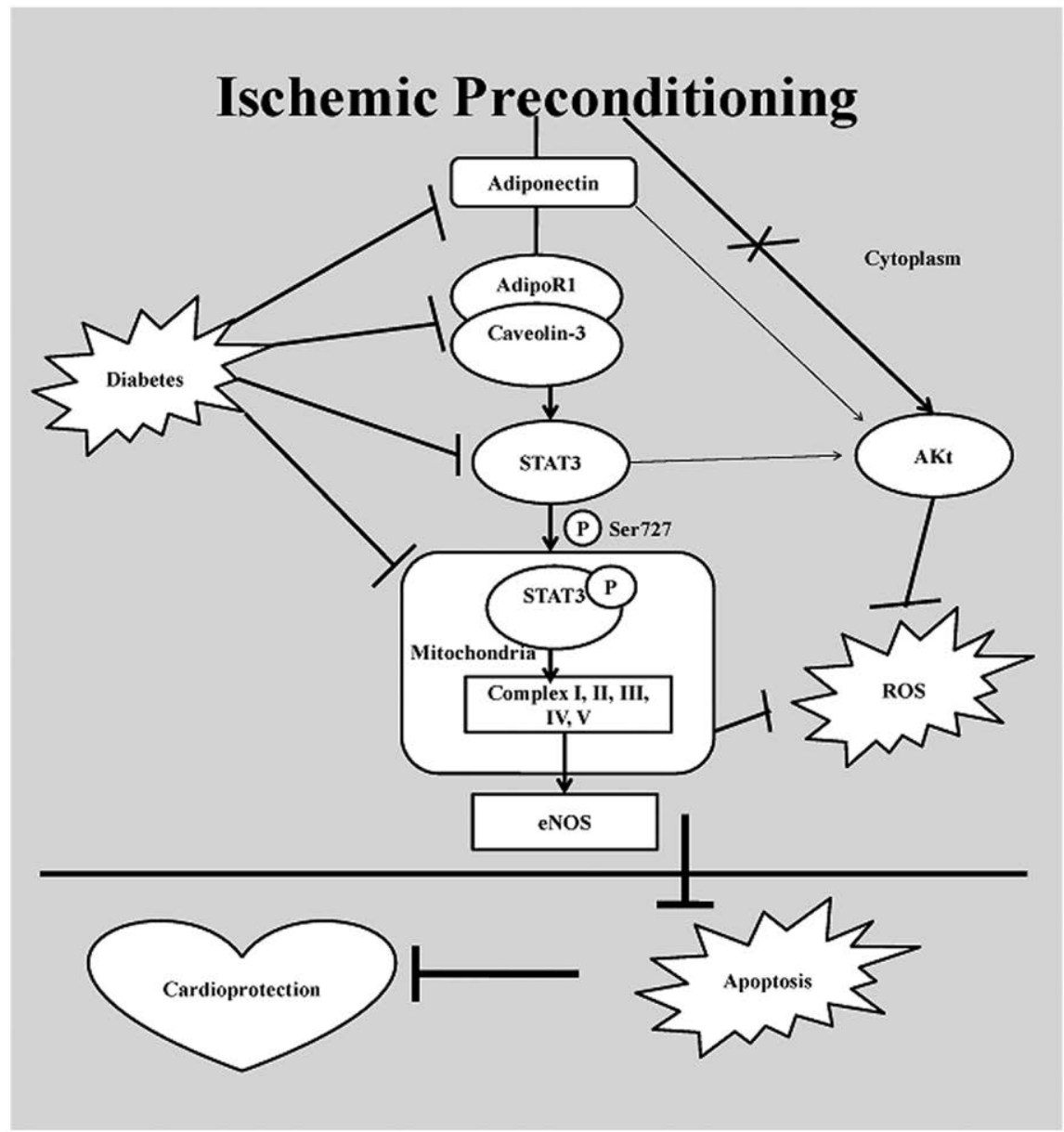

- Fig. 3 Schematic of proposed signaling engaged with APN-intervened mitoSTAT3 actuation IPo cardioprotection under nondiabetic and diabetic conditions. Under nondiabetic condition, IPo presents cardioprotective impacts through associatively upregulating cardiomyocytes APN articulation and improving the communication of AdipoR1 with Cav3, prompting the initiation of STAT3, which in this way translocates into mitochondria and upgrades mitochondrial complex I/II + III/IV/V exercises. These, together with IPo-interceded actuation of Akt, result in diminished myocardial oxidative pressure and weakened cardiomyocyte apoptosis and in the long run lessen IR damage. Nonetheless, under diabetic condition, IPo neglects to actuate STAT3 due to the lessened cardiomyocyte APN creation and disabled AdipoR1 and Cav3 connection. These, together with the failure of IPo to enact Akt, prompt poor postischemic mitochondrial work, bringing about improved myocardial oxidative pressure and expanded cardiomyocyte apoptosis.

cav-1 show constricted microvascular sequestration of neutrophils and lung damage in response to compare with intraperitoneal lipopolysaccharide (LPS) [74]. In this model, cav-1, by means of direction of eNOS-inferred nitric oxide production, appears to be a crucial determinant of NF-B enactment and ensuing pulmonary infection because of LPS. There is additionally confirm that cav- 1 assumes a key part in antigen-presenting cells, prompting actuation of T lymphocytes [75], and in regulating macrophage activation.

Connection of testosterone with nitric oxide level through the activity of caveolin

Testosterone deficiency has been involved in the pathophysiology of heart failure, adding to a few attributes of this disorder, for example, lessened skeletal mass, oxygen utilization, decreased exercise limit and cachexia [76]. Gonadectomy in male rats changed the transcriptional and translational control of gene encoding the L-type calcium $\left(\mathrm{Ca}^{2+}\right)$ channel, the $\mathrm{Na}+/ \mathrm{Ca} 2+$ exchanger, $\beta 1$ adreno- ceptors, and myosin dark chain subunits which lessened cardiomyocyte contractile capacity [77].

There are a many proposed work through which testosterone may follow up on NO synthesis and their release. An investigation from 2012 demonstrated that testosterone, by means of nongenomic activation of intracellular signaling pathways and $\mathrm{Ca}^{2+}$ influx, increments endothelial NO synthesis and moreover represses platelet aggregation [78]. Moreover, in another examination where vascular ageing was investigated, testosterone increased expression of gene that control replicative life span which in this way inhibited endothelial senescence by means of upregulation of eNOS activity [79]. Testosterone insufficiency may prompt lessening NO release by make complex of caveolin and eNOS. Caveolin, the caveolar membrane protein is a negative controller of eNOS, as its between activity and binding restricting the activity of eNOS by making caveolin-eNOS complex [80]. Caveolin keeps up eNOS in inactivated state, which prompts diminish in NO generation [81]. 
Connection of ischaemic preconditioning through the activity of caveolin

The Ischaemic preconditioning (IPC) boost has been demonstrated to prompt 2 particular windows of cardioprotection. The primary window happens without a moment's delay after the IPC boost and keeps going 2-3 h (named 'established IPC' or 'intense IPC'), after which the effect melts away and vanishes. The second window takes after 12-24 $\mathrm{h}$ later, and endures 48-72 $\mathrm{h}$ (named 'deferred IPC' or 'second window of assurance') [82].

Current document is Daidzein restores the attenuated cardioprotective effect of IPC in ovariectomized rat heart, which may additionally be due to downregulation of caveolin that leads to elevated availability of $\mathrm{NO}$ and consequent increase in the activation of mitochondrial $K_{\text {ATP }}$ channels. The result show that perfusion of L-NAME, an eNOS inhibitor, and glibenclamide, a $\mathrm{K}_{\text {ATP }}$ channel blocker, significantly attenuated the DDZ-induced cardioprotective impact of IPC in ovariectomized [83] and diabetic rat heart [13]. Gupta et al. mentioned that activation of Heme oxygenase-1 via a specific activator, that is, hemin, restored the attenuated cardioprotective effect of IPC in diabetic rat heart by means of disrupting the caveolin-eNOS complicated and thereby enhancing the release of NO. When pretreatment with Zinc protoporphyrin, a particular Heme oxygenase- 1 inhibitor drastically blocked the restoration of cardioprotective impact of IPC in Heme pretreated diabetic rat heart. Therefore, it exhibit that cardioprotective effect of IPC in diabetic rat heart is due to reduced activity of Heme oxygenase- 1 and due to this fact reduced undertaking of eNOS [84].

\section{Conclusion}

A lot of essential research in cell science and signaling pathway investigation has been done, concerning about the structure and capacity of caveolae. Caveolae show to a stable membrane developed around caveolin and cholesterol-rich space in a Golgi apparatus. A physiological role of Cav-1 in the direction of eNOS activation and proves evidence that the support of Cav-1 and eNOS could be a novel target site for the control of cardiovascular disorder and other various diseases. It seems that the impact of caveolin on cellular signaling depends upon the setting a particular cell finds itself in. Down-regulation of Cav-1 role in these cells, by interfering of RNA sequencing, it reduced their tumorigenic and metastatic potential, apart from this Cav-1 articulation result in extended apoptosis, with extended prostate apoptosis reaction factor- 4 and PTEN levels in Cav-1(-/ - ) invalid prostate tumor. Studies of caveolin have implicated these structures in a host of human diseases, including diabetes, cancer, cardiovascular disease, atherosclerosis, pulmonary fibrosis, and a variety of degenerative muscular dystrophies. To provide a base for clinically applicable therapeutic strategies Furthermore, research in this area need to validate the possible interaction of caveolin or caveolae in the pathology of various diseases.

\section{Clinical implications}

It has been indicated from various disorders that caveolin are critical which trigger IPC as opposed to caveolin membrane. The increment in caveolin mediations decides metabolic changes and energy required within the dissipation.
Caveolin can be determined with a role in pharmacological and biophysical properties. Nevertheless, the importance of the caveolin reconsidered in cardio-protection in present findings. The usefulness of such studies indicates a fundamental role of caveolin in a variety of CVS disorders including arrthymias, hypertension and heart failures.

These findings suggest that caveolin within the adjusting ischemic-reperfusion injury in rat. The heart rate of the mouse occurred during ischemia and probably enhances vigilant extrapolation applied to larger warm blooded animals.

These discoveries recommend that caveolin find contributory role in regulating ischemic-reperfusion injury in mice and more responsible to open surgery of higher animals and human hearts.

\section{Limitations of study}

A study of mechanistic pathways mediating caveolin provides the knowledge of cell functions and cardio protection and limits to cardiovascular functions and pathways. Limitations of this study include missing of experimental protocols with justification and involvement of caveolin. A lot of advancements in this field those are not proved till date which are not included. A lack of wide versatility of caveolin in the study for physiological functions.

\section{Acknowledgement}

All authors contributed equally to this work. The authors are thankful to the Integral University \& KNIMT-FOP for providing facilities, critical suggestion regarding the improvement of the manuscript and special thanks to research community of Integral University for providing manuscript no. IU/R\&D/2018-MCN-000448.

\section{Conflict of Interest}

None.

\section{References}

[1] Pilch PF, Meshulam T, Ding $S$ et al. Caveolae and lipid trafficking in adipocytes. Clin Lipidol 2011; 6: 49-58

[2] Yamada $E$. The fine structure of the gall bladder epithelium of the mouse. J Biophys Biochem Cytol 1955; 1: 445-458

[3] Palade GE. Fine structure of blood capillaries. J Appl Physics 1953; 24: $14-24$

[4] Thomas CM, Smart EJ. Caveolae structure and function. J Cell Mol Med 2008; 12: 796-809

[5] Thorn H, Stenkula KG, Karlsson M et al. Cell surface orifices of caveolae and localization of caveolin to the necks of caveolae in adipocytes. Mol Biol Cell 2003; 14: 3967-3976

[6] Moos T. Brain iron homeostasis. Dan Med Bull 2002; 49: 279-301

[7] Kiss AL, Turi A, Muller N et al. Caveolae and caveolin isoforms in rat peritoneal macrophages. Micron 2002; 33: 75-93

[8] Balijepalli RC, Kamp T]. Caveolae, ion channels and cardiac arrhythmias. Prog biophys Mol Biol 2008; 98: 149-160

[9] Parton RG, Simons K. The multiple faces of caveolae. Nat Rev Mol Cell Biol 2007; 8: 185-194 
[10] Smart E], Graf GA, McNiven MA et al. Caveolins, liquid-ordered domains, and signal transduction. Mol Cell Biol 1999; 19: 7289-7304

[11] Halayko AJ, Stelmack GL. The association of caveolae, actin, and the dystrophin-glycoprotein complex: A role in smooth muscle phenotype and function? Can J Physiol Pharmacol 2005; 83: 877-891

[12] Das M, Das DK. Caveolae, caveolin, and cavins: Potential targets for the treatment of cardiac disease. Ann Med 2012; 44: 530-541

[13] Ajmani P, Yadav HN. Possible involvement of caveolin in attenuation of cardioprotective effect of ischemic preconditioning in diabetic rat heart. BMC Cardiovasc. Disord 2011; 11: 43

[14] Machleidt T, Li WP, Liu P et al. Multiple domains in caveolin-1 control its intracellular traffic. J Cell Biol 2000; 148: 17-28

[15] Lisanti MP, Tang ZL, Sargiacomo M. Caveolin forms a hetero-oligomeric protein complex that interacts with an apical GPI-linked protein: implications for the biogenesis of caveolae. J Cell Biol 1993; 123 : 595-604

[16] Uittenbogaard A, Smart EJ. Palmitoylation of caveolin-1 is required for cholesterol binding, chaperone complex formation, and rapid transport of cholesterol to caveolae. J Biol Chem 2000; 275: 25595-25599

[17] Vinten J, Voldstedlund M, Clausen $\mathrm{H}$ et al. A 60- kDa protein abundant in adipocyte caveolae. Cell Tissue Res 2001; 305: 99-106

[18] Jansa P, Mason SW, Hoffmann-Rohrer U et al. Cloning and functional characterization of PTRF, a novel protein which induces dissociation of paused ternary transcription complexes. EMBO J 1998; 17: 2855-2864

[19] Hasegawa T, Takeuchi A, Miyaishi O et al. PTRF (polymerase I and transcriptrelease factor) is tissue-specific and interacts with the BFCOL1 (binding factor of a type-I collagen promoter) zinc-finger transcription factor which binds to the two mouse type-I collagen gene promoters. Biochem J 2000; 347: 55-59

[20] Vinten ], Johnsen AH, Roepstorff P et al. Identification of a major protein on the cytosolic face of caveolae. Biochim Biophys Acta 2005; 1717: $34-40$

[21] Chang W], Rothberg KG, Kamen BA et al. Lowering the cholesterol content of MA104 cells inhibits receptor-mediated transport of folate. J Cell Biol 1992; 118: 63-69

[22] Thyberg J, Calara F, Dimayuga P et al. Role of caveolae in cholestero transport in arterial smooth muscle cells exposed to lipoproteins in vitro and in vivo. Lab Invest 1998; 78: 825-837

[23] Hailstones D, Sleer LS, Parton RG et al. Regulation of caveolin and caveolae by cholesterol in MDCK cells. J Lipid Res 1998; 39: 369-379

[24] Smart EJ, Ying YS, Conrad PA et al. Caveolin moves from caveolae to the Golgi apparatus in response to cholesterol oxidation. J Cell Biol 1994; 127: 1185-1197

[25] Li S, Couet ], Lisanti MP. Src tyrosine kinases, Galpha subunits, and $\mathrm{H}$-Ras share a common membrane-anchored scaffolding protein, caveolin. Caveolin binding negatively regulates the auto-activation of Src tyrosine kinases. J Biol Chem 1996; 271: 9182-29190

[26] Schlegel A, Lisanti MP. The caveolin triad: caveolae biogenesis, cholesterol trafficking, and signal transduction. Cytokine Growth Factor Rev 2001; 12: 41-51

[27] Arnal JF, Dinh-Xuan AT, Pueyo M et al. Endothelium-derived nitric oxide and vascular physiology and pathology. Cell Mol Life Sci 1999; 55: 1078-1087

[28] Andrew PJ, Mayer B. Enzymatic function of nitric oxide synthases. Cardiovasc Res 1999; 43: 521-531

[29] Shaul PW. Endothelial nitric oxide synthase, caveolae and the development of atherosclerosis. J Physiol 2003; 547: 21-33

[30] Balligand JL, Kobzik L, Han X et al. Nitric oxide-dependent parasympathetic signaling is due to activation of constitutive endothelia (type III) nitric oxide synthase in cardiac myocytes. J Biol Chem 1995; 270: 14582-14586
[31] Garcia-Cardena G, Martasek P, Masters BS et al. Dissecting the interaction between nitric oxide synthase (NOS) and caveolin. Functional significance of the nos caveolin binding domain in vivo. J Biol Chem 1997; 272: 25437-2544

[32] Campbell L, Gumbleton M, Ritchie K. Caveolae and the caveolins in human disease. Physiol Rev 2004; 84: 1341-1379

[33] Bender SB, Jia G, Sowers JR. Mineralocorticoid receptors: An appealing target to treat coronary microvascular dysfunction in diabetes. Diabetes 2015; 64: 3-5

[34] Seto SW, Krishna SM, Yu H et al. Impaired acetylcholine-induced endothelium-dependent aortic relaxation by caveolin-1 in angiotensin II-infused apolipoprotein-E (ApoE-/-) knockout mice. PLoS One 2013; 8: e58481

[35] Sharma A, Sellers S, Stefanovic N et al. Direct endothelial nitric oxide synthase activation provides atheroprotection in diabetes-accelerated atherosclerosis. Diabetes 2015; 64: 3937-3950

[36] Jia G, Sowers JR. Caveolin-1 in cardiovascular disease: A double-edged sword. Diabetes 2015; 64: 3645-3747

[37] Breton S, Lisanti MP, Tyszkowski R et al. Basolateral distribution of caveolin-1 in the kidney. Absence from $\mathrm{H}+$-atpase-coated endocytic vesicles in intercalated cells. J Histochem. Cytochem 1998; 46: 205-214

[38] Wang L, Zhang C, Su X et al. Caveolin-1 defciency inhibits the basolateral $\mathrm{K}+$ channels in the distal convoluted tubule and impairs renal k+ and Mg2 + transport. J Am Soc Nephrol 2015; 26: 2678-2690

[39] Paunescu TG, Lu HA, Russo LM et al. Vasopressin induces apical expression of caveolin in rat kidney collecting duct principal cells. Am J Physiol Renal Physiol 2013; 305: F1783-F1795

[40] Chen Z, Bakhshi FR, Shajahan AN et al. Nitric oxide-dependent Src activation and resultant caveolin-1 phosphorylation promote eNOS/ caveolin-1 binding and eNOS inhibition. Mol Biol Cell 2012; 23 : 1388-1398

[41] Hanahan D, Weinberg RA. Hallmarks of cancer: the next generation. Cell 2011; 144: 646-674

[42] Williams TM, Hassan GS, Li J et al. Caveolin-1 promotes tumor progression in an autochthonous mouse model of prostate cancer: genetic ablation of Cav-1 delays advanced prostate tumor development in tramp mice. J Biol Chem 2005; 280: 25134-15145

[43] Quann K, Gonzales DM, Mercier I et al Caveolin-1 is a negative regulator of tumor growth in glioblastoma and modulates chemosensitivity to temozolomide. Cell Cycle 2013; 12: 1510-1520

[44] McLaughlin N, Annabi B, Bouzeghrane M et al. The Survivin-mediated radioresistant phenotype of glioblastomas is regulated by RhoA and inhibited by the green tea polyphenol (-)-epigallocatechin-3-gallate. Brain Res 2006; 1071: 1-9

[45] Shatz M, Liscovitch M. Caveolin-1: a tumor-promoting role in human cancer. Int J Radiat Biol 2008; 84: 177-189

[46] Yang G, Truong LD, Wheeler TM et al. Caveolin-1 expression in clinically confined human prostate cancer: a novel prognostic marker. Cancer Res 1999; 59: 5719-5723

[47] Ho CC, Huang PH, Huang HY et al. Up-regulated caveolin-1 accentuates the metastasis capability of lung adenocarcinoma by inducing filopodia formation. Am J Pathol 2002; 161: 1647-1656

[48] Joo HJ, Oh DK, Kim YS et al. Increased expression of caveolin1 and microvessel density correlates with metastasis and poor prognosis in clear cell renal cell carcinoma. BJU Int 2004; 93: 291-296

[49] Kato K, Hida Y, Miyamoto M et al. Overexpression of caveolin-1 in esophageal squamous cell carcinoma correlates with lymph node metastasis and pathologic stage. Cancer 2002; 94: 929-933

[50] Couet], Sargiacomo M, Lisanti MP. Interaction of a receptor tyrosine kinase, EGF-R, with caveolins. Caveolin binding negatively regulates tyrosine and serine/threonine kinase activities. J Biol Chem 1997; 272: 30429-30438 
[51] Eldar-Finkelman H, Krebs EG. Phosphorylation of insulin receptor substrate 1 by glycogen synthase kinase 3 impairs insulin action. Proc Natl Acad Sci USA 1997; 94: 9660-9664

[52] Yamamoto M, Toya Y, Jensen RA et al. Caveolin is an inhibitor of platelet-derived growth factor receptor signaling. Exp Cell Res 1999; 247: $380-388$

[53] Ishikawaa Y, Otsua K, Oshikawa J. Caveolin; different roles for insulin signal? Cell Signal 2005; 17: 1175-1182

[54] Zagoory O, Braiman A, Priel Z. The mechanism of ciliary stimulation by acetylcholine: Roles of calcium, PKA, and PKG. J Gen Physiol 2002; 119: 329-339

[55] Wessler IK, Kirkpatrick C]. The non-neuronal cholinergic system: An emerging drug target in the airways. Pulm Pharmacol Ther 2001; 14 : 423-434

[56] Konig P, Dedio J, Oess S et al. NOSIP and its interacting protein, eNOS, in the rat trachea and lung. J Histochem Cytochem 2005; 53: 155-164

[57] Krasteva G, Pfeil U, Drab M et al. Caveolin-1 and -2 in airway epithelium: Expression and in situ association as detected by FRET-CLSM. Respir Res 2006; 7: 108

[58] Li C, Duan W, Yang F et al. Caveolin-3-anchored microdomains at the rabbit sarcoplasmic reticulum membranes. Biochem Biophys Res Commun 2006; 344: 1135-1140

[59] Krasteva G, Pfeil U, Filip AM et al. Caveolin-3 and eNOS colocalize and interact in ciliated airway epithelial cells in the rat. Int J Biochem Cell Biol 2007; 39: 615-625

[60] McNally EM, de Sa Moreira E, Duggan D] et al. Caveolin-3 in muscular dystrophy. Hum Mol Genet 1998; 7: 871-877

[61] Gazzerro E, Sotgia F, Bruno C et al. Caveolinopathies: from the biology of caveolin-3 to human diseases. Eur J Hum Genet 2010; 18: 137-145

[62] Bruning JC, Michael MD, Winnay JN et al. A muscle-specific insulin receptor knockout exhibits features of the metabolic syndrome of NIDDM without altering glucose tolerance. Mol Cell 1998; 2: 559-569

[63] Eldar-Finkelman H, Schreyer SA, Shinohara MM et al. Increased glycogen synthase kinase-3 activity in diabetes- and obesity-prone C57BL/6] mice. Diabetes 1999; 48: 1662-1666

[64] Nandi A, Kitamura Y, Accili D. Mouse models of insulin resistance. Physiol Rev 2004; 84: 623-647

[65] Cohen AW, Combs TP, Scherer PE et al. Role of caveolin and caveolae in insulin signaling and diabetes. Am J Physiol Endocrinol Metab 2003; 285: $1151-1160$

[66] Dupuy AM, Mas E, Ritchie K et al. The relationship between apolipoprotein E4 and lipid metabolism is impaired in Alzheimer's disease. Gerontology 2001; 47: 213-218

[67] Corder EH, Saunders AM, Strittmatter W] et al. Gene dose of apolipoprotein E type 4 allele and the risk of Alzheimer's disease in late onset families. Science 1993; 261: 921-923
[68] Hyman BT, West HL, Rebeck GW et al. Quantitative analysis of senile plaques in Alzheimer disease: observation of log-normal size distribution and molecular epidemiology of differences associated with apolipoprotein E genotype and trisomy 21 (Down syndrome). Proc Natl Acad Sci USA 1995; 92: 3586-3590

[69] Premkumar DR, Cohen DL, Hedera P et al. Apolipoprotein E-epsilon4 alleles in cerebral amyloid angiopathy and cerebrovascular pathology associated with Alzheimer's disease. Am J Pathol 1996; 148: 2083-2095

[70] Poirier J, Davignon J, Bouthillier D et al. Apolipoprotein E polymorphism and Alzheimer's disease. Lancet 1993; 342: 697-699

[71] Simons K, Ikonen E. Functional rafts in cell membranes. Nature 1997; 387: $569-572$

[72] Galbiati F, Razani B, Lisanti MP. Emerging themes in lipid rafts and caveolae. Cell 2001; 106: 403-411

[73] Gaudreault SB, Dea D, Poirier J. Increased caveolin-1 expression in Alzheimer's disease brain. Neurobiol Aging 2004; 25: 753-759

[74] Garrean S, Gao XP, Brovkovych V et al. Caveolin-1 regulates NF-kappaB activation and lung inflammatory response to sepsis induced by lipopolysaccharide. J Immunol 2006; 177: 4853-4860

[75] Ohnuma K, Inoue H, Uchiyama M et al. T-cell activation via CD26 and caveolin-1 in rheumatoid synovium. Mod Rheumatol 2006; 16: 3-13

[76] Malkin C], Channer KS, Jones TH. Testosterone and heart failure. Curr Opin Endocrinol Diabetes Obes 2010; 17: 262-268

[77] Golden KL, Marsh JD, jiang Y et al. Gonadectomy alters myosin heavy chain composition in isolated cardiac myocytes. Endocrine 2004; 24: 137-140

[78] Campelo AE, Cutini PH, Massheimer VL. Testosterone modulates platelet aggregation and endothelial cell growth through nitric oxide pathway. J Endocrinol 2012; 213: 77-87

[79] Ota H, Akishita M, Akiyoshi T et al. Testosterone deficiency accelerates neuronal and vascular aging of SAMP8 mice: protective role of eNOS and SIRT1. PLoS One 2012; 7: e29598

[80] Marsh JD, Lehmann MH, Ritchie RH et al. Androgen receptors mediate hypertrophy in cardiac myocytes. Circulation 1998; 98: 256-261

[81] Feron O, Dessy C, Moniotte $S$ et al. Hypercholesterolemia decreases nitric oxide production by promoting the interaction of caveolin and endothelial nitric oxide synthase. J Clin Invest 1999; 103: 897-905

[82] Marber MS, Latchman DS, Walker JM et al. Cardiac stress protein elevation 24 hours after brief ischemia or heat stress is associated with resistance to myocardial infarction. Circulation 1993; 88: 1264-1272

[83] Goyal A, Semwal BC, Yadav HN. Abrogated cardioprotective effect of ischemic preconditioning in ovariectomized rat heart. Hum Exp Toxicol 2016; 35: 644-653

[84] Gupta I, Goyal A, Singh NK et al. Hemin, a heme oxygenase-1 inducer, restores the attenuated cardioprotective effect of ischemic preconditioning in isolated diabetic rat heart. Hum Exp Toxicol 2017; 36: 867-875 